\title{
Interrelation between Climate and Dengue in Malaysia
}

\author{
Bryan Paul1,2,3*, Wai Liang Tham ${ }^{4}$ \\ ${ }^{1}$ Department of Biological Sciences, University of Alberta, Edmonton, Canada \\ ${ }^{2}$ Department of Earth and Atmospheric Sciences, University of Alberta, Edmonton, Canada \\ ${ }^{3}$ Department of Renewable Resources, University of Alberta, Edmonton, Canada \\ ${ }^{4}$ Department of Biochemistry and Molecular Biology, University of British Columbia, Vancouver, Canada \\ Email: ${ }^{*}$ bpaul@ualberta.ca
}

Received 21 April 2015; accepted 8 June 2015; published 11 June 2015

Copyright (C) 2015 by authors and Scientific Research Publishing Inc.

This work is licensed under the Creative Commons Attribution International License (CC BY).

http://creativecommons.org/licenses/by/4.0/

(c) (i) Open Access

\begin{abstract}
Dengue cases in Malaysia are on the rise and have worsened since last decade, and this has generally been attributed to human actions. However, the effects of climate in this situation have been under-mentioned. We argue that climate also plays a role in spreading dengue transmission and multiple studies have shown that climate and transmission of infectious diseases are closely interconnected. Our evaluation examines how local climate influences dengue transmission by studying two parameters, specifically local average surface temperature and average precipitation, and we assume that a changing climate will influence the number of reported dengue cases and mortality rates. We also study the potential impact of climate change on the transmission of dengue and its distribution over a large geographical region, and have found that dengue and infectious diseases in general tend to be widespread in regions with higher or increasing surface temperature.
\end{abstract}

\section{Keywords}

\section{Climate, Dengue, Malaysia}

\section{Introduction}

\subsection{Global Climate Observation}

Current climate models predict that global surface temperatures may experience increases of up to $8^{\circ} \mathrm{C}$ and an increase in carbon dioxide $\left(\mathrm{CO}_{2}\right)$ concentration up to $1000 \mathrm{ppmv}$ by 2100 [1] [2]. This increase will not only influence the future climate, but also other environmental systems which we depend on. Besides that, such ${ }^{*}$ Corresponding author. 
changes can alter the behavior of infectious diseases and pose global health concerns [3]. The World Health Organization (WHO) noted that if the earth experiences a fast pace of climate change, the transmission of infectious diseases will follow a similarly rapid trend, which in time could eventually become the sole cause of disease transmission [4].

\subsection{Malaysia Dengue Overview}

Located in equatorial Southeast Asia, Malaysia provides a suitable environment for infectious diseases to spread all year-round [5]. Dengue in particular has a tremendous effect on the livelihood of communities in Malaysia. Recent statistics released by Malaysia's Ministry of Health (MOH) shows that Malaysia experienced a 59\% increase in the number of cases reported in January 2015 compared to similar period in 2014 [6]. Out of all the number of cases reported, 44 proved fatal [6]. The $\mathrm{MOH}$ added that these numbers are increasing monthly, and as of February 2015, 23,966 cases have already been reported this year with 62 fatalities. Figure 1 illustrates the number of cases and mortality rates reported from January 2014 to February 2015. Overall rising trends are observed in both shown datasets and it is predicted that the trend will continue in the near future. In addition, the WHO findings placed Malaysia on a list of Southeast Asian and Western Pacific regions with a high number of cases of reported dengue [6].

\subsection{Climate as Drivers}

Many studies posit human behaviors as the primary driving force for the rising trend observed in the reported number of cases. Factors such as population growths, increased infrastructure, increased agricultural activities, overcrowding, and sanitization are often associated with increased frequencies of dengue transmission in the community [7]. In addition, increased travelling and shipping networks also play some role in distributing dengue locally and globally [8]-[10]. As for climate change, a study by Abdul Rahman (2012) argued that climate change is most likely a minor player in the spread of dengue due to poor correlations between climate and historical incidence. However, Banu et al. (2011) argued that the poor correlation might be due to lag-phases experienced during the transitions between local climate cycles.

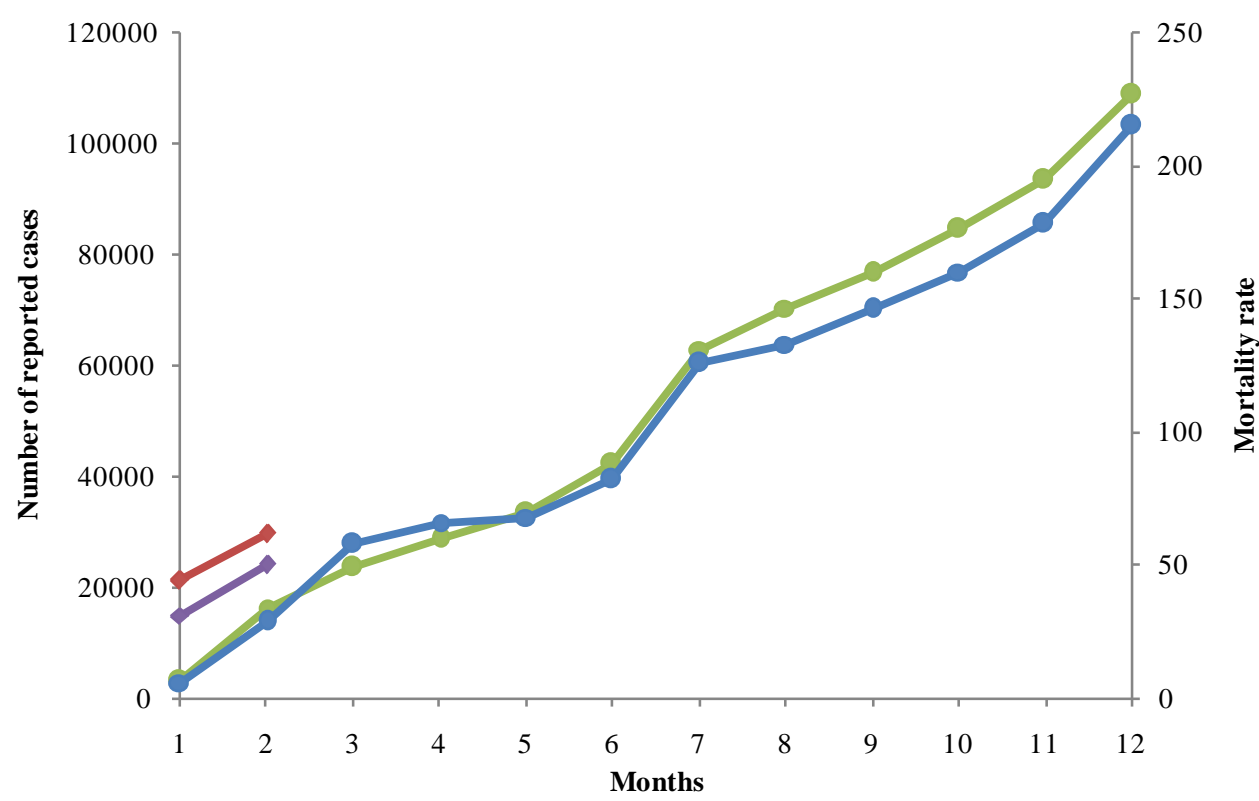

Figure 1. Reported dengue cases and mortality rates in Malaysia from January 2014 to February 2015. The green line represents the number of cases reported in 2014, the purple line the number of cases in 2015, the blue line the number of mortalities in 2014, and the red line the number of mortalities in 2015. An overall rising trend is shown for both parameters. In addition, a sharp spike is observed between the months of June and July 2014 in both the number of cases and mortality rates. In 2015, the number of reported cases jumps in the order of thousands compared with the same period in 2014, with the mortality rate showing the same trend [6]. 
We tried evaluating the role of climate in the transmission of dengue by using data from reported cases in 2014 and data which was made available from an international climate agency. Our goal is to find a correlation between climate and dengue cases. Two parameters, in this case average surface temperature and average precipitation, are used as proxies for climate. Since historical data is not available from the local meteorological department, we resorted to using historical datasets provided by the US National Climatic Data Center (NCDC). We utilized three out of five different meteorological stations (MSs) in Malaysia as provided by the NCDC (partly due to one of the MS's lack of complete data, while another MS had no data from 1995 onwards)to measure the average temperature and precipitation (Figure 2). These MSs are the Setiawan MS, the Kuching MS, and the Sandakan MS. These three MSs cover large geographical areas in Malaysia and provide a relatively good estimate of the country local surface temperature and precipitation. Statistical evaluation on monthly temperatures and precipitation are illustrated in Table 1.

\section{Analysis}

We first examined correlational data to investigate the relationship between the monthly average surface temperatures and the number of reported cases (Figure 3(a)). The monthly average surface temperature from three MSs for year 2014 is $23.7^{\circ} \mathrm{C}\left(n=12, \sigma^{2}=0.34, \sigma_{\bar{x}}=0.58, R^{2}=0.2448\right)$. We observed no particular overall trends in compared datasets but we did however observe two distinct relationships in the months of February and May. It is shown that in February, when the average surface temperature dropped, the number of reported cases increased, and vice-versa in May. We have also observed a rising linear trend in the average temperature profile where $\left(R^{2}=0.2248\right)$. However, the result obtained differs from most available literature since studies conducted have all concluded that dengue spreads fairly well at higher temperatures and not the other way around [15]. Carrington et al. (2013) added that dengue vector, Aedes aegypti, is particularly competent when the temperature ranges from $20^{\circ} \mathrm{C}$ to $30^{\circ} \mathrm{C}$. We also noted that there is a transition between the northeast monsoon and southwest monsoon in May, when local temperature and precipitation distribution in Malaysia is affected [12]. The northeast monsoon is often associated with cold surges while the southwest monsoon causes warm ones [12].

Next, we set up the correlation to investigate the relationship between the monthly average precipitation and number of reported cases in 2014 (Figure 3(b)). It is recorded that the average precipitation for 2014 from all three MSs is at $177.5 \mathrm{~mm}\left(n=12, \sigma^{2}=12690.1, \sigma_{\bar{x}}=112.7, R^{2}=7 E^{-05}\right)$. There are no particular overall trends for both datasets tested, similar to the observation in Figure 3(a). However, we managed to distinguish two sets of trends, one for the months of February to March as well as September to December. Both sets show a distinct

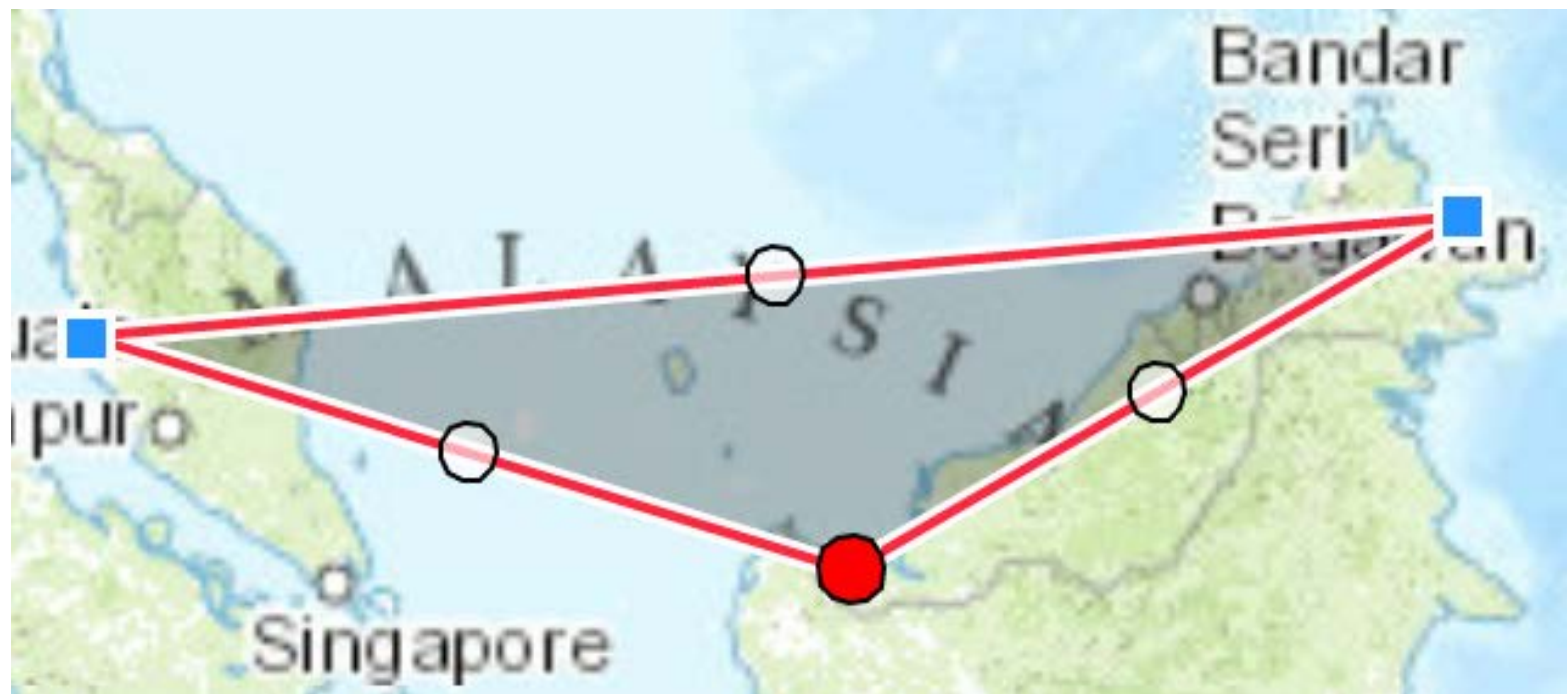

Figure 2. A triangular grid representing the geographical area covered by meteorological stations in Setiawan, Kuching, and Sandakan. These particular stations are selected because data from them is available from the NCDC. The triangular grid encompasses one location on the peninsula and two locations in East Malaysia (on the island of Borneo). The average area coverage within the triangular grid is approximately 400,000 $\mathrm{km}^{2}$. Map image and grid utilized ArcGIS software [23]. 
Table 1. Statistical evaluation on monthly surface temperatures and precipitation measured at three meteorological stations in Malaysia. Datasets are provided by the NCDC [11].

\begin{tabular}{|c|c|c|c|c|c|c|c|c|c|c|c|c|c|}
\hline \multicolumn{2}{|c|}{ Month } & Jan & Feb & Mar & Apr & May & Jun & Jul & Aug & Sept & Oct & Nov & Dec \\
\hline \multirow{2}{*}{ Average } & ${ }^{\circ} \mathrm{C}^{\mathrm{a}}$ & 22.7 & 22.5 & 23.7 & 24.1 & 24.4 & 24.3 & 23.9 & 23.6 & 23.8 & 23.9 & 23.9 & 23.9 \\
\hline & $\mathrm{mm}^{\mathrm{b}}$ & 452.0 & 184.9 & 28.1 & 97.3 & 111.4 & 96.6 & 94.8 & 224.8 & 122.4 & 153.2 & 223.8 & 310.6 \\
\hline \multirow{2}{*}{$\begin{array}{l}\text { Standard } \\
\text { Deviation }\end{array}$} & Temp. & 0.31 & 0.35 & 0.36 & 0.61 & 0.17 & 0.55 & 0.10 & 0.35 & 0.35 & 0.56 & 0.44 & 0.46 \\
\hline & Prec. & 317.99 & 171.55 & 73.44 & 153.12 & 66.94 & 87.10 & 125.00 & 192.07 & 62.12 & 111.54 & 51.52 & 191.84 \\
\hline \multirow{2}{*}{ Error } & Temp. & 0.18 & 0.20 & 0.21 & 0.35 & 0.10 & 0.32 & 0.06 & 0.20 & 0.20 & 0.32 & 0.25 & 0.27 \\
\hline & Prec. & 183.59 & 99.04 & 42.40 & 88.40 & 38.65 & 50.29 & 72.17 & 110.89 & 35.86 & 64.40 & 29.75 & 110.81 \\
\hline
\end{tabular}

ANOVA single factor test.

\begin{tabular}{ccccccccc}
\hline Source of Variation & Conditions & SS & $\boldsymbol{d} \boldsymbol{f}$ & $\boldsymbol{M S}$ & $\boldsymbol{F}$ & $\boldsymbol{P}$-values & $\boldsymbol{F}$ crit & Note \\
\hline \multirow{2}{*}{ Between Group } & Temp. & 2.317 & 2 & 1.1586 & 2.981 & 0.065 & 3.285 & Null hypothesis accepted $^{\mathrm{c}}$ \\
& Prec. & 116722.7 & 2 & 58361.33 & 2.235 & 0.123 & 3.285 & Null hypothesis accepted $^{\mathrm{d}}$ \\
Within Group & Temp. & 12.856 & 33 & 0.3887 & & & & \\
& Prec. & 861598.2 & 33 & 26109.04 & & & & \\
Total & Temp. & 15.143 & 35 & & & & & \\
& Prec. & 978320.9 & 35 & & & & & \\
\hline
\end{tabular}

${ }^{a}$ Original values are measured in degrees Fahrenheit and converted to the centigrade scale. ${ }^{\mathrm{b}}$ Original values are measured in inches and converted to the meter scale. ${ }^{\mathrm{c}} \mathrm{H}_{\mathrm{o}}=$ Temperature influenced the rising number of dengue reported cases in Malaysia. ${ }^{\mathrm{d}} \mathrm{H}_{\mathrm{o}}=$ Precipitation influenced the rising number of dengue reported cases in Malaysia.
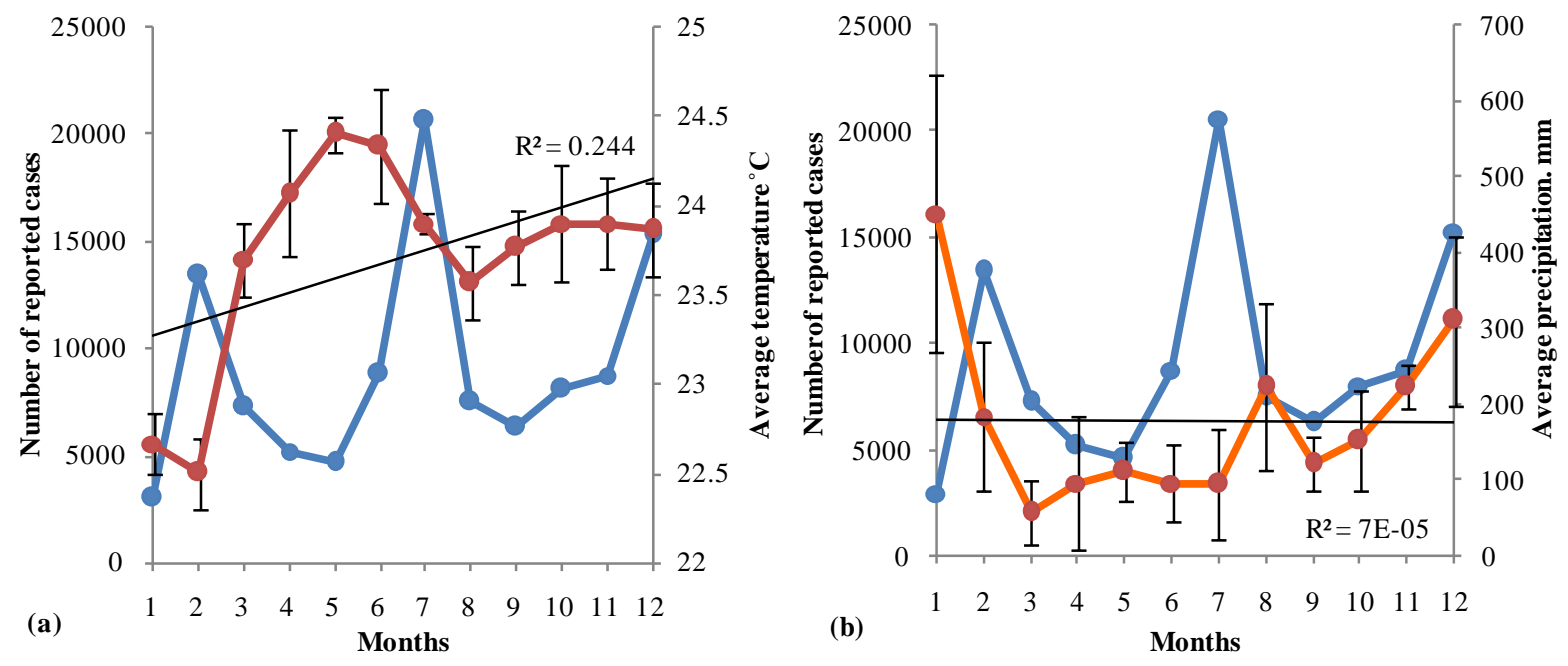

Figure 3. (a) Number of reported dengue cases and average temperature in Setiawan, Kuching, and Sandakan MSs; (b) Number of reported dengue cases and average monthly precipitation in Setiawan, Kuching, and Sandakan MSs. The red line represents the average monthly temperature while the two blue lines represent the number of reported cases each month. The orange line represents the average monthly precipitation.

lag after months when heavy precipitation occurred. Banu et al. (2011) explained this by stating that numerous studies have shown similar lag-phase behavior between the transmission of dengue after a rainy period and these lag-phases can be attributed to numerous biological factors. We have also observed a small rising trend $\left(R^{2}=7 E^{-05}\right.$ ) in average precipitation, but it is negligible and unlikely to contribute significantly towards the spread of dengue. A study conducted by Thu et al. (1998) [14] observed that the developmental cycle of dengue normally peaks during the rainy season and slowly decreases towards the dry monsoon period. It is also noted 
that dengue is positively correlated with increased rainfall since outbreaks normally occur within this period [15]. We noted that the heavy precipitation-causing northeast monsoons are predominant during this period [10]. Conversely, Malaysia experienced an intense drought in February and March, which explains the huge drop in average precipitation in Figure 3(b) [16].

Using the correlations obtained for average surface temperatures, average precipitation, and the number of monthly reported cases, we then proceed to infer how climate change might influence the future transmission of dengue in Malaysia. Although our results do not show the intended positive correlation between temperature and precipitation in increasing dengue transmission, we assume that with the predicted increase in global temperatures, the number of reported dengue cases would continue to fluctuate depending on how global warming affects regional and local climate. Our predictions of a fluctuating number of cases are supported by an argument from McMichael et al. (2006) [17] where although an increased surface temperature might support the growth of dengue, the availability of watersheds for vector reproduction will ultimately decide whether it can continue its transmission cycle. Furthermore, we also noted the impacts of climate change on modifying the strength and periodicity of local monsoons. Since monsoons are thought to influence dengue transmission, climate change can offset and weaken these monsoons through time by influencing local temperature variation and precipitation, and by extension affecting the transmission of the disease [18].

\section{Future Predictions}

With the increase in global temperatures, the transmission of dengue can spread across larger global geographical region (Figure 4). Our view is augmented from Abdul Rahman's (2012) and Patz et al. studies (2003) [20], which they state that climate change has the capacity to directly affect the potential transmission rate of infectious disease and spread them over large terrestrial areas. De Souza et al. (2012) added that climate change can cause the migration of infectious diseases into newer regions and this can lead to safety hazards for local communities while inducing immunological stresses in sterile individuals. In Figure 4, it is illustrated that the future transmission area of the similar mosquito-borne disease malaria is expected to surpass the current longitudinal boundaries tropical and sub-tropical zones by 2050. Dengue, which is similar to malaria for mode of transmission,

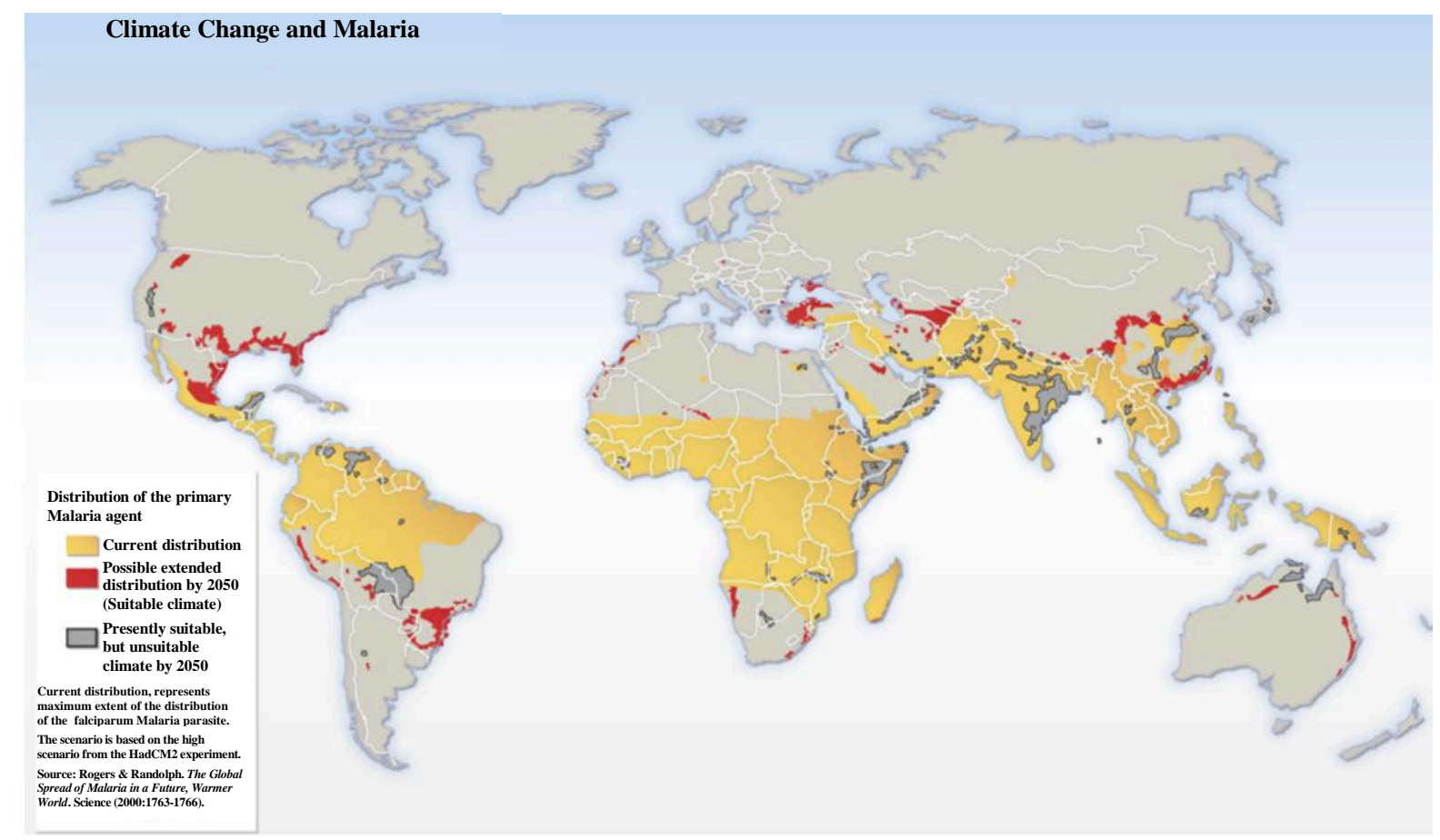

Figure 4. Geographical areas currently and expected to be affected by Malaria. Yellow-shaded regions represent the current distribution of Malaria vectors worldwide. Red-shaded regions represent the possible extent of Malaria distribution by 2050 following climate change. Dengue which has similar mode of transmission as Malaria is expected to follow the distribution observed. Figure adapted from De-Souza et al. (2012) of which they utilized UNEP's Global Risk Data Platform [19]. 
is infectious and competent within a temperature range from $16^{\circ} \mathrm{C}$ to $38^{\circ} \mathrm{C}$ [13]. With the predicted increase of global surface temperatures within the next century, regions bordering Malaysia and other dengue-infected regions might experience increased dengue transmission.

\section{Discussion}

Although future cases of dengue transmission are difficult to predict, it is important to note that there are mathematical models that can be used to study the global transmission of infectious diseases. The utilization of these models will not only help Malaysia to restrict the prevalence of dengue, but they can also help local hospitals and clinics prepare for the outburst of a dengue endemic during certain times in the year. Keeling and Danon (2009) [21] show that the current mathematical models used to model infectious diseases with climate change as a parameter are solid and can be utilized to predict the future transmission of any particular infectious diseases. In addition, modeling often helps clinicians to prepare for any major epidemics and allows intervention planning in the case of a disease outbreak [22]. However, it is argued that due to the complexity and dynamics of vectors and hosts, mathematical models can only provide limited information that renders them far from being accurate.

Our analysis provides details of the influence of climate on the rising number of dengue reported cases in Malaysia. We provide a small scale climate-disease analysis that spans a year, but this is insufficient to pinpoint climate as major cause in the recent increase in dengue transmission. However, promising data regarding the influence of climate change on monsoon seasons allow us to we predict that with the projected increase in climate variation, transmission of dengue in Malaysia will also change. Unfortunately, Malaysia lacks the information needed to provide a full-scale analysis of the impact of climate on dengue transmission, since both the $\mathrm{MOH}$ and the Malaysian Meteorological Department did not have databases of previous datasets made available for public. We recommend that more studies to be conducted in order to better understand the influence of climate on dengue transmission in Malaysia, as well the setting-up of data bases by government agencies to store past information on climate and health-related statistics which will prove invaluable in conducting future research.

\section{Acknowledgements}

The authors wish to acknowledge the datasets provided by NCDC for the purpose of this study. These datasets of Malaysia's meteorological stations can be obtained from the following site

(http://www.ncdc.noaa.gov/cdo-web/datasets/GHCNDMS/locations/FIPS:MY/detail). We would like to thank ArcGIS: ESRI for allowing us to use their software to triangulate and calculate total area coverage from our three meteorological stations. In addition, we would also like to thank the Malaysian Meteorological Department for granting us full access to their website. This study is conducted by undergraduates and co-authored by Bryan Paul and Wai Liang Tham. Editing and proof-reading carried out by Wai Liang Tham.

\section{References}

[1] McCarthy, J.J., et al. (Eds.) (2001) Climate Change 2001: Impacts, Adaptation, and Vulnerability: Scenario of the 21st Century. IPCC, Cambridge.

[2] Zickfeld, K., Morgan, M.G., Frame, D.J. and Keith, D.W. (2010) Expert Judgements about Transient Climate Response to Alternative Future Trajectories of Radiative Forcing. Proceedings of the National Academy of Sciences, 107, 12451-12456. http://dx.doi.org/10.1073/pnas.0908906107

[3] Ostfeld, R.S. and Brunner, J.L. (2015) Climate Change and Ixodes Tick-Borne Diseases of Humans. Philosophical Transactions of the Royal Society, 370, 1-11.

[4] McMichael, A.J. (Eds.) (2003) Climate Change and Human Health—Risks and Responses. WHO, Geneva.

[5] Cheah, W.K., et al. (2014) A Review of Dengue Research in Malaysia. Medical Journal of Malaysia, 69, 59-67.

[6] (2015) Emerging Disease Surveillance Response: Dengue Situation Update 435-460. WHO, Geneva.

[7] Abdul Rahman, H. (2012) Climate Change and Dengue Fever Diseases: Any Association? International Scientific Journal, 2, 1-5.

[8] Mangold, K. and Reynolds, S. (2013) Review of Dengue Fever. Pediatric Emergency Care, 29, 665-669. http://dx.doi.org/10.1097/PEC.0b013e31828ed30e

[9] Wongkoon, S., Jaroensutasinee, M. and Jaroensutasinee, K. (2013) Distribution, Seasonal Variation \& Dengue Trans- 
mission Prediction in Sisaket, Thailand. Indian Journal of Medical Research, 138, 347-353.

[10] Colon-Gonzalez, F.J., Fezzi, C., Lake, I.R. and Hunter, P.R. (2013) The Effects of Weather and Climate Change on Dengue. PLOS Neglected Tropical Diseases, 7, 1-9. http://dx.doi.org/10.1371/journal.pntd.0002503

[11] NCDC (2015) Monthly Climatological Summary (2014). NCDC, North Carolina.

[12] Malaysian Meteorological Department (2015) General Climate of Malaysia. Malaysian Meteorological Department, Kuala Lumpur.

[13] Carrington, L.B., Veronica Armijos, M., Lambrechts, L., Barker, C.M. and Scott, T.W. (2013) Effects of Fluctuating Daily Temperatures at Critical Thermal Extremes on Aedes aegypti Life-History Traits. PLoS ONE, 8, e58824. http://dx.doi.org/10.1371/journal.pone.0058824

[14] Thu, H.M., Aye, K.M. and Thein, S. (1998) The Effect of Temperature and Humidity on Dengue Virus Propagation in Aedes aegypti Mosquitos. Southeast Asian Journal of Tropical Medicine and Public Health, 2, 280-284.

[15] Banu, S. Hu, W.B., Hurst, C. and Tong, S.L. (2011) Dengue Transmission in the Asia-Pacific Region: Impacts of Climate Change and Socio-Environmental Factors. Tropical Medicine \& International Health, 16, 598-607. http://dx.doi.org/10.1111/j.1365-3156.2011.02734.x

[16] Chong, P.K. and Pakiam, R. (2014) Water Rationing Begins outside Malaysia Capital amid Drought. In Bloomberg Business. http://www.bloomberg.com/news/articles/2014-02-25/water-rationing-begins-outside-malaysia-s-capital-amid-drought

[17] McMichael, A.J., Woodruff, R.E. and Hales, S. (2006) Climate Change and Human Health: Present and Future Risk. The Lancet, 367, 859-869. http://dx.doi.org/10.1016/S0140-6736(06)68079-3

[18] Loo, Y.Y., Billa, L. and Singh, A. (2014) Effect of Climate Change on Seasonal Monsoon in Asia and Its Impact on the Variability of Monsoon Rainfall in Southeast Asia. Geoscience Frontiers, in press.

[19] de Souza, D.K., Owusu, P.N. and Wilson, M.D. (2012) Impact of Climate Change on the Geographic Scope of Diseases. InTech, Morn Hill, 245-264.

[20] Patz, J.A., et al. (2003) Chapter 6: Climate Change and Infectious Diseases. Climate Change and Human Health: Risks and Responses, WHO, Geneva.

[21] Keeling, M.J. and Danon, L. (2009) Mathematical Modelling of Infectious Diseases. British Medical Bulletin, 92, 3342. http://dx.doi.org/10.1093/bmb/ldp038

[22] Rodó, X., Pascual, M., Doblas-Reyes, F.J., Gershunov, A., Stone, D.A., et al. (2013) Climate Change and Infectious Diseases: Can We Meet the Need for Better Prediction? Climatic Change, 118, 625-640. http://dx.doi.org/10.1007/s10584-013-0744-1

[23] (2015) Malaysia Map. ArcGIS: ESRI, Redlands, USA. 